\title{
DAD e LEAD. Nuove forme di partenariato tra sistema educativo-formativo e famiglie
}

\author{
Grazia Romanazzi
}

\begin{abstract}
In emergenza pandemica da nuovo coronavirus, l'umanità intera è stata chiamata a raccolta affinché ognuno assumesse l'impegno di dare il proprio prezioso contributo al contenimento del contagio, per tutelare la salute di tutti. Il tributo pagato da ciascuno è stato elevatissimo in termini economici, sociali e relazionali. Molti hanno pagato con la propria vita. In campo educativo, i LEAD (Legami Educativi A Distanza) e la DAD (Didattica A Distanza) hanno tentato di "ridurre il danno e l'impatto" del distanziamento sociale a carico degli alunni, rispettivamente degli asili nido e della scuola dell'infanzia, i primi; della scuola primaria e secondaria, la seconda. I limiti oggettivi dettati dalla carenza e/o inadeguatezza dei mezzi, degli strumenti e dei materiali a disposizione in casa, o altrimenti reperibili, in tempo di lockdown hanno rappresentato inconfutabili criticità tanto per gli alunni e le loro famiglie, quanto per gli insegnanti. Questi ultimi sono stati, sovente, soggetti ad accuse di pretenziosità sproporzionata rispetto alle reali possibilità contingenti di ciascun allievo. Le famiglie, dal canto loro, hanno dovuto confrontarsi con una "prossimità" abitativa, emotiva e relazionale tra i vari componenti, alla quale i tempi lavorativi le avevano disabituate. Il presente contributo intende superare le accuse e le forme di reciproco ostracismo tra sistemi educativo-formativi e famiglie per sottolineare, di contro, l'imprescindibilità di forme inedite di partenariato. Solo la collaborazione tra gli agenti sociali consente infatti di oltrepassare l'isolamento e l'individualismo in favore di una comunità educante alla resilienza, al processo e al divenire, più che al risultato.
\end{abstract}

Parole chiave: Didattica A Distanza (DAD), Legami Educativi A Distanza (LEAD), Covid-19, insegnanti, scuole chiuse.

\begin{abstract}
During the new coronavirus pandemic, each of us was called upon to contribute to limit the infection and protect everyone's health. All of us paid a very high price, in economic, social and relationship terms. Many people paid with their life. In the educational field, "distance educational ties" and remote teaching have attempted to reduce the demage of social distancing on students, from nursery to secondary school. During the lockdown, teachers and pupils were affected by the lack and the inadequasy of the means, tools
\end{abstract}

${ }^{1}$ Cultrice della materia in Pedagogia generale e sociale presso il Dipartimento di Scienze della Formazione, dei Beni Culturali e del Turismo dell'Università degli Studi di Macerata. 
and materials available. Teachers have often been accused of demanding too much from scholars. Families, on the other side, faced a housing, emotional and relational proximity they were not accustomed to. This contribution aims to overcome mutual accusations and highlight the importance of new forms of partnership between school and families. Only the collaboration between social agents allows to overcome isolation and individualism and to facilitate a community able to educate to resilience, to the learning process and to grow up, rather than to a single result.

Keywords: remote teaching, "distance educational ties”, Covid-19, teachers, closed schools.

\section{Introduzione}

L'inizio del 2020 sarà ricordato alla stregua di una nuova "guerra fredda" a causa dell'incombenza dell'emergenza pandemica da Covid-19, a cui l'umanità intera ha pagato un tributo elevatissimo, sia nei termini delle vite stroncate dal virus SARS-CoV-2, sia in termini di sacrifici e rinunce, necessari per non soccombere alla minaccia di una vera catastrofe epidemiologica.

L'8 marzo 2020, il Presidente del Consiglio dei Ministri della Repubblica italiana, Giuseppe Conte, ha annunciato il temuto lockdown: la sospensione di tutte le attività commerciali e produttive, la chiusura di tutti i centri di aggregazione e finanche dei luoghi di culto, il divieto di assembramenti e l'obbligo di distanziamento sociale. Le strade si sono svuotate, le luci sono state spente, il mondo si è fermato. Le conseguenze di questo brusco e repentino arresto del sistema globale hanno e, plausibilmente, avranno una portata devastante: pensiamo al tracollo economico di aziende piccole e medie, che non riescono più a sostentarsi autonomamente e che, con l'allentamento delle misure restrittive, faticano a ripartire; alla difficoltà e alle perdite di mercato delle grandi imprese, che hanno dovuto porre i propri dipendenti in cassa integrazione; ancora, alla disperazione dei lavoratori in nero o atipici, delegittimati dal rivendicare qualsivoglia diritto e costretti a ricorrere alle - esigue - misure di protezione e assistenza sociale appositamente promulgate dallo Stato.

\section{I bambini, al tempo del Covid: $i$ "grandi assenti" dalle politiche nazionali}

Il prezzo da pagare è stato altissimo anche sul piano umano: si tratta dell'isolamento di persone a cui è stato prescritto l'obbligo di restare a casa: luogo non sempre felice, talvolta inospitale, angusto, sovraffollato da presenze abitualmente transitanti e raramente stanziali o, all'inver- 
so, prigione di solitudini (Corsi, Stramaglia, 2009). Basti pensare agli anziani soli, alle famiglie separate, monogenitoriali e con figlio unico, ai single (Corsi, Stramaglia, 2010). Eppure, lo scotto maggiore l'hanno forse pagato i grandi assenti dalle politiche e dai decreti d'emergenza, i dimenticati dai piani di ripartenza: i bambini.

Il 5 marzo 2020 è stata disposta la sospensione delle attività didattiche e la chiusura delle scuole di ogni ordine e grado sul territorio nazionale. I bambini si sono ritrovati, improvvisamente, a casa, a stretto contatto con gli altri componenti familiari, con i quali, in precedenza, condividevano, al più, i pasti e i fine settimana. «La modernità di fine secolo ha abbreviato i tempi di empatia fra genitori e figli, e viceversa, e diradato assai le occasioni di un mutuo godimento d'intimità affettiva e spirituale» (Galli, 2000, p. 194).

D'un tratto, i bambini e i ragazzi, "normalizzati” all'assenza fisica e/o psicologica dei genitori dediti al lavoro extradomestico (ivi, pp. 192-202), hanno potuto godere - o soffrire, in taluni casi - della presenza costante e quotidiana di quelli, in una «epifania delle relazioni familiari» (Scabini, Cigoli, 2000, p. 60), che ne ha svelato la struttura, la qualità, «i suoi punti di forza e di debolezza, il suo essere fonte di costruzione o decostruzione della persona» (Ibidem). Inizialmente è sembrata una inaspettata, quanto gradita, vacanza. Finché non è sopraggiunta la consapevolezza che qualcosa di importante stava accadendo e cambiando le vite di tutti: non è più stato possibile andare a scuola, incontrare i propri amici, interagire con educatori e insegnanti. I più piccoli, al riguardo, necessitano di spiegazioni puntuali per comprendere motivazioni e implicazioni complesse.

In ottemperanza al diritto alla libertà di espressione e all'informazione, chiara e comprensibile, sancito dall'art. 13 della Convenzione ONU sui diritti dell'infanzia e dell'adolescenza del 1989: «È importante parlare ai bambini e ai ragazzi dell'emergenza sanitaria che sta coinvolgendo il nostro paese e il modo intero, utilizzando un linguaggio semplice e adeguato alla loro capacità cognitiva ed emotiva» (MIUR, AGIA, 2020, p. 4). A tal fine, abbiamo assistito al dilagare di filmati di bambini e personaggi fantastici che spiegano cos'è il coronavirus, quali comportamenti adottare per evitarne la diffusione e l'importanza, al proposito, di restare a casa. I bambini, a ogni buon conto, percepiscono e vivono a proprie spese gli impedimenti di incontrare nonni e zii, di giocare e imparare con i compagni di classe, di correre al parco, di non avere più l'agenda settimanale colma di impegni sportivi e sociali. Il tempo dei piccoli, così come quello degli adulti - dotati, invero, di capacità e strumenti di informazione e comprensione ben diversi - è stato sospeso. È un tempo 
che non c'è perché non più scandito nei momenti specifici delle routine quotidiane, ma che esiste ed è grevissimo nella sua lungaggine e inabitabilità sociale e condivisa.

L'emergenza coronavirus ha caratteristiche nuove e, oltre a coinvolgere la sfera sanitaria, colpisce la sfera psicologica ed emotiva di tutti, anche delle persone di minore età, con conseguenze negative soprattutto per i più fragili. L'obiettivo, in questo momento di veri e propri stravolgimenti, è certamente quello di non sconvolgere totalmente i ritmi della loro quotidianità, ma anche quello di educare i bambini e i ragazzi a rispondere flessibilmente ai cambiamenti, adattandovisi e traendo da essi la capacità di dare risposte creative e innovative. In tal modo, l'emergenza sanitaria sarà stata l'occasione per dare a tutti una possibilità di apprendimento: imparare che è possibile trarre insegnamento anche dalle difficoltà, trasformando un momento di crisi in risorsa (ivi, pp. 2-3).

La chiarezza e l'esaustività delle informazioni fornite rispondono, dunque, non solo al bisogno dei più piccoli di essere ascoltati e di partecipare attivamente ai processi decisionali e di sviluppo del contesto di appartenenza, ma anche al diritto di «godere del miglior stato di salute possibile» (Convenzione sui diritti dell'infanzia e dell'adolescenza, art. 24, p. 13). Offrire la possibilità di esprimere, contenere e rassicurare le ansie, le paure e le incertezze cagionate dall'attuale stile di vita contribuisce al benessere psicologico del bambino e dell'adolescente in un'imprescindibile visione olistica degli stessi e, nello specifico, del loro stato di salute. È importante elaborare e superare la paura, valicare simbolicamente i confini dell'isolamento coatto.

Per passare, in tal modo, da una "crisi come dissoluzione", che non ci è estranea e che potrebbe essere quasi "dietro l'angolo", a una "crisi come ridecisione”. Virtuosa, morale, individuale, di gruppo, di contesto. Politica, nel significato più nobile del termine. Quella crisi che, nella sua etimologia, rinvia alla "scelta" e al conseguente superamento (proposto e voluto) di un evento traumatico e stressante o di una situazione, al presente, instabile e pericolosa (Corsi, 2012, p. 215).

\section{Scuola e servizi per l'infanzia di fronte alla sfida pandemica: DAD e LEAD come possibili promotori di "prossimità" didattico-educativa}

Con l'intento di fronteggiare l'emergenza e di superare la crisi, a seguito della chiusura delle scuole e della sospensione delle attività didatti- 
che in presenza, disposte con il Dpcm del 4 marzo 2020, è stata avviata e intensificata, per la scuola primaria e secondaria, la Didattica a Distanza (DAD), oggetto di numerose e, talvolta, feroci critiche rivolte agli insegnanti da parte di genitori colti alla sprovvista, spiazzati, disabituati e impreparati alla costante prossimità fisica, emotiva e relazionale con i propri figli. La finalità nobile della Didattica a Distanza è di ricreare, in un ambiente di apprendimento virtuale, la scuola come comunità educante di persone e pratiche in inter e cor-relazione: «luogo in cui crescere sani, responsabili, competenti. [...] un ambiente di apprendimento che facilita la relazione educativa, la condivisione, il piacere di conoscere, la creatività e il benessere. Si cresce tutti, insieme: ragazzi e adulti» (Avanguardie Educative, 2020, https://www.indire.it/wp-content/themes/indire-2018/manifesto-la-scuola-non-si-ferma.pdf).

I genitori, molti dei quali già oberati dalle difficoltà di gestione dello smartworking, si sono trovati nella contingenza di aiutare i figli a districarsi tra le video-lezioni e i compiti, spesso con mezzi e strumenti tecnologici inadeguati o insufficienti, una connessione internet precaria e molti altri limiti materiali e contestuali. Sono, dunque, genitori esausti, che hanno dovuto rispolverare le proprie reminiscenze scolastiche, laddove presenti, e, soprattutto, "combattere" contro la resistenza dei figli a rispondere con responsabilità e puntualità alle richieste dei docenti, seppure a distanza. I genitori hanno spesso accusato gli insegnanti di assegnare troppi compiti, di inviare troppo materiale da stampare, di non compenetrarsi nell'oggettiva difficoltà di adempiere a tutte le richieste, specie in mancanza degli adeguati supporti software e hardware. Sono state, per lo più, ignorate o trascurate le non poche criticità dell'attività di insegnamento a distanza, nel tentativo di proseguire e perseguire una continuità curricolare e relazionale, in linea con la programmazione ministeriale e la progettualità educativa, che nulla improvvisa. Nondimeno, il corpo docente ha dovuto far fronte a una scarsità di mezzi, materiali e strumenti nell'ambiente domestico parimenti limitante e frustrante.

Nello specifico della prima infanzia, la Commissione Infanzia Sistema Integrato Zero-Sei (D.lgs. 65/2017) ha optato per i LEAD: Legami Educativi a Distanza, al fine di rimarcare la natura affettiva e relazionale del rapporto educativo che si instaura nei contesti dell'asilo nido e della scuola dell'infanzia. Lungi da uno stile di insegnamento-apprendimento performativo e performante, i LEAD non intendono dettare istruzioni precise per l'esecuzione corretta di compiti che esercitino specifiche abilità e competenze; al più, essi mirano a veicolare un'educazione profonda che sviluppi l'identità, stimoli l'autonomia, favorisca l'acquisi- 
zione delle competenze sociali e rinsaldi il senso di reciprocità, corresponsabilità, comunità e appartenenza di ciascuno. La finalità fondante l'ideologia sottesa a questo ambizioso e lungimirante progetto, nato in situazione di emergenza - è bene ricordarlo - si declina su un duplice versante: nell'immediato, è importante ricreare, anche a distanza, il legame di emozioni, sguardi, voci, complicità, "prossimità umana" che, fino a pochi mesi fa, alimentava la quotidianità scolastica dei bambini; con lo sguardo prospettico della postura pedagogica che ci è peculiare, non possiamo esimerci dal progettare, poi, con speranza e fiducia, forme inedite dei futuri legami "in presenza", nuove modalità di partecipazione e costruzione di significati comuni, che prenderanno forma in contesti organizzativi ripensati nell'ottica di un "graduale ritorno alla normalità", la quale sarà, inevitabilmente, diversa da quella ante-Covid.

I LEAD, grazie all'uso avveduto e mirato dei mezzi tecnologici più diffusi, non di rado tacciati di fuorviare l'attenzione e la formazione dei bambini e dei giovani di oggi, si costruiscono in ambiente virtuale, ovvero in uno spazio-tempo esposto all'anonimia e all'indeterminatezza identitaria, relazionale e storica dei «nonluoghi surmoderni» descritti da Marc Augé (1992, trad. it. 1993, passim).

L'ambiente virtuale è intangibile, non ha confini, non si può esplorare con il corpo e il movimento, non consente il contatto fisico, l'abbraccio, la coccola, gesti essenziali in misura inversamente proporzionale all'età dei bambini, ma ha potenzialità diverse, che sfruttano soprattutto i canali visivo e uditivo, e può offrire stimoli per esplorare l'ambiente fisico attraverso gli altri sensi, e, al pari di qualsiasi altro ambiente, ha delle regole di comportamento [Commissione Infanzia Sistema Integrato Zero-Sei (cfr. D.lgs. 65/2017, p. 2)].

Pertanto, è necessario che siano gli educatori e gli insegnanti a connotare umanamente l'ambiente virtuale, al fine di renderlo un luogo realmente formativo. È necessario, in tal senso, rinnovare il partenariato che sancisce l'alleanza tra scuola e famiglie. Non è ipotizzabile, tantomeno auspicabile, lasciare il bambino piccolo solo dinanzi al mezzo tecnologico prescelto o disponibile per fruire dei LEAD. È indispensabile la presenza e la mediazione di un genitore, cui si chiede di collaborare per stabilire e rispettare appuntamenti e tempi di collegamento, reperire materiali, predisporre attività e supportare la prole nella partecipazione circolare e collettiva a quanto proposto dal corpo docente. Ravvisiamo, nel patto educativo tra genitori e insegnanti così rimodulato, le tracce della "gestione sociale” del nido e della scuola dell'infanzia che Enzo Catarsi (2008, cfr. 
in particolare le pp. 109-116) riconduceva a Loris Malaguzzi e a Bruno Ciari: il coinvolgimento delle famiglie rappresentava, negli anni Sessanta del Novecento, oltre che un processo di decentramento e democratizzazione dello Stato, la preziosa opportunità di una conoscenza "ecologica" dei bambini, a partire dalla storia personale di ciascuno e dal contesto sociale di appartenenza, specificato dalle e nelle dinamiche intra-familiari.

Modernamente, il riferimento corre a un modello "partecipato" di scuola. Catarsi intravedeva, con lungimiranza, un ulteriore salto di qualità nel sostegno alla genitorialità, per una più profonda comprensione delle paure e delle ansie genitoriali e, conseguentemente, dei bambini, emergenti dai racconti di questi. Basti pensare ai dubbi e al bisogno di confronto, specie dei neogenitori, sulle principali tappe delle diverse aree di sviluppo: autonomia nel mangiare, nel vestirsi e nello spogliarsi, acquisizione del controllo sfinterico, abbandono del succhietto, sospensione dell'allattamento materno ecc. Si tratta di un'intuizione di grande attualità, oggi che c'è uno sconfinamento della scuola negli ambienti domestici degli alunni, e viceversa. La modalità di connessione a distanza più frequentemente utilizzata è, infatti, la video-chiamata o video-lezione, che rende visibili scorci di intimità ed espone a un reciproco giudizio altrimenti precluso: gli educatori possono avere modo di osservare gli stili educativi genitoriali; i genitori, a loro volta, possono avere l'opportunità di osservare le dinamiche e le modalità relazionali degli insegnanti con i loro figli e con il gruppo classe.

È importante, dunque, dotarsi vicendevolmente di delicatezza e discrezione; fornire e chiedere feedback rispetto alle attività svolte e ai risultati raggiunti, specie nei termini dell'acquisizione di autonomia; adeguare l'offerta formativa a distanza a eventuali richieste specifiche, per renderla quanto più personalizzata possibile, nel rispetto della programmazione e della contingenza situazionale. Nello specifico della fascia d'età 0-6, il gioco e l'interazione tra pari rappresentano i punti di forza di ogni attività proposta e mai imposta: sono pertanto utili, a ragion veduta, le video-chiamate e i lavori in piccoli gruppi, il montaggio di filmati, la ricostruzione di storie, il canto corale, la ricomposizione di un puzzle, alternando e unendo il contributo di ciascun bambino.

\section{Conclusioni}

Alla luce di quanto fin qui affermato, urge dunque sottolineare l'importanza che "nessuno resti indietro": pertanto, pur nella difficoltà di 
raggiungere alcune famiglie deprivate o svantaggiate tecnologicamente, culturalmente, socialmente ed economicamente, gli educatori e gli insegnanti stanno utilizzando, e potranno utilizzare, ogni risorsa o "aggancio" possibile.

Il lavoro e lo sforzo umano, oltre che professionale, compiuto oggi più che mai da una classe insegnante troppo spesso bistrattata, sottostimata e delegittimata, è davvero ingente ed encomiabile. Non dimentichiamo che si tratta, anche nel loro caso, pur sempre di persone, recluse in casa, spaventate e preoccupate da un virus sconosciuto, come chiunque altro. A loro, tuttavia, viene richiesto di tenere le redini del mondo formativo dell'infanzia e giovanile, altrimenti alla deriva, dando prova di presenza di spirito e immediatezza di risposte, testimoniando speranza e infondendo fiducia nella scuola e nel futuro: ancora possibili, ancora condivisibili. Genitori e insegnanti sono, da ambo le parti, provati da un'emergenza sanitaria che mai si sarebbe potuto immaginare di vivere. Non è tempo di accusarsi e osteggiarsi reciprocamente; al contrario, questo è il tempo della "resistenza" collettiva e condivisa.

Oggi, sostenuti e incoraggiati dall'intera comunità educante, i bambini e i ragazzi imparano la lezione più importante di tutte: con l'esempio virtuoso e la condotta responsabile di ciascuno, è possibile cambiare l'esistenza di molti e salvare la vita di qualcuno. Domani, superata questa dura prova, in sinergia e nella comunanza di intenti, avremo educato $\mathrm{i}$ giovani alla resilienza.

\section{Riferimenti bibliografici}

Augé M. (1992): Nonluoghi. Introduzione a una antropologia della surmodernità. Trad. it. Milano: Elèuthera, 1993.

Avanguardie Educative (2020): Manifesto della scuola che non si ferma (https:// www.indire.it/wp-content/themes/indire-2018/manifesto-la-scuola-non-siferma.pdf; data di ultima consultazione: 24.5.20).

Catarsi E. (2008): Pedagogia della famiglia. Roma: Carocci.

Commissione Infanzia Sistema Integrato Zero-Sei (D.lgs. 65/2017) (2020): Orientamenti pedagogici sui LEAD: Legami Educativi A Distanza. Un modo diverso per fare nido e scuola dell'infanzia (https://www.miur.gov.it/ documents/20182/2432359/Sistema+zero_sei+orientamenti+pedagogici. $\mathrm{pdf} / 3 \mathrm{~b} 0$ ea 542 - a $8 \mathrm{bf}-3965-61 \mathrm{f} 0-453$ e 85 ae $87 \mathrm{~d} 5$ ? version $=1.0$ $\& \mathrm{t}=1589880921017$; data di ultima consultazione: 26.5 .20 ).

Convenzione sui diritti dell'infanzia e dell'adolescenza (1989): https://www.datocms-assets.com/30196/1602516091-convenzionedirittiinfanzia.pdf (data di ultima consultazione: 6.2 .21 ). 
Corsi M., Stramaglia M. (2009): Dentro la famiglia. Pedagogia delle relazioni educative familiari. Roma: Armando Editore.

Corsi M., Stramaglia M. (2010): Il fine politico dell'educazione familiare. In L. Carrera (a cura di): Fare o non fare politica. Soggetti, modi e luoghi. Milano: Guerini.

Corsi M. (2012): Oltre il vuoto della nuova Babele: lo "sguardo obliquo". In M. Stramaglia (a cura di): Pop pedagogia. L'educazione postmoderna tra simboli, merci e consumi. Lecce-Brescia: Pensa MultiMedia, pp. 203-217.

Galli N. (2000): Pedagogia della famiglia ed educazione degli adulti. Milano: Vita e Pensiero.

Ministero dell'Istruzione, Autorità garante per l'infanzia e l'adolescenza (2020): Didattica a distanza e diritti degli studenti. Mini-guida per docenti (https:// www.istruzione.it/coronavirus/allegati/miniguida_mi_AGIA_6_4_2020_. pdf; data di ultima consultazione: 24.5.20).

Scabini E., Cigoli V. (2000): Il famigliare. Legami, simboli e transizioni. Milano: Raffaello Cortina. 